REVISTA

Actualidades Investigativas en Educación http://revista.inie.ucr.ac.cr/

ISSN 1409-4703

\title{
INVESTIGACIONES DE LAS REPRESENTACIONES SOCIALES DEL MEDIO AMBIENTE EN BRASIL Y MÉXICO
}

RESEARCHES OF SOCIAL REPRESENTATIONS OF ENVIRONMENTAL IN BRAZIL AND MEXICO

\section{Volumen 13, Número 1}

Enero - Abril

pp. $1-20$

Este número se publicó el 30 de enero de 2013

Raúl Calixto Flores

Revista indizada en REDALYC, SCIELO

Revista distribuida en las bases de datos:

CATÁLOGO DE LATINDEX, IRESIE, CLASE, DIALNET, DOAJ, E-REVIST@S, SHERPA/ROMEO, QUALIS

Revista registrada en los directorios:

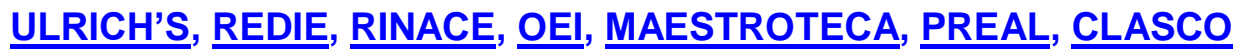




\title{
INVESTIGACIONES DE LAS REPRESENTACIONES SOCIALES DEL MEDIO AMBIENTE EN BRASIL Y MÉXICO \\ RESEARCHES OF SOCIAL REPRESENTATIONS OF ENVIRONMENTAL IN BRAZIL AND MEXICO
}

\begin{abstract}
Resumen: En este artículo se describen algunas investigaciones sobre las representaciones sociales del medio ambiente en Brasil y en México, con el objetivo de observar las implicaciones de estos estudios para la educación ambiental; destaca el hecho de que sus resultados son coincidentes, a pesar de que sean distintas culturas y lenguajes, así como diferentes grupos de población y niveles educativos. La exploración de las investigaciones muestra la pertinencia del empleo de la teoría de las representaciones sociales, para identificar, comprender y explicar el pensamiento social en el campo de la educación ambiental. Los autores más citados en las investigaciones anteriormente referidas son Denise Jodelet, Serge Moscovici, Marcos Reigota y Jean Claude Abric. Durante esta breve revisión, se observa el predominio de las investigaciones realizadas con estudiantes, la mayoría utiliza una metodología cualitativa y un enfoque interpretativo. En las diversas investigaciones analizadas, sobre las representaciones del medio ambiente, se evidencia su importancia para comprender la práctica de la educación ambiental. Por ende, para la educación ambiental resulta fundamental comprender las formas en que se establecen las representaciones sociales, en la desconstrucción-construcción de los elementos que forman su pensamiento cotidiano. Considerando los resultados de las investigaciones revisadas, se puede afirmar que el medio ambiente es un objeto social complejo, cultural y contextualmente determinado.
\end{abstract}

Palabras clave: EDUCACION, AMBIENTE, INVESTIGACIÓN, REPRESENTACIONES SOCIALES, MÉXICO, BRASIL

\begin{abstract}
In this article we describe some researches on social representations of the environment in Brazil and Mexico, with the aim to observe the implications of the studies for the environmental education; highlights the fact that their results are coincident, although they are different cultures and languages, and different population groups and educational levels. The exploration of the research shows the relevance of using the theory of social representations, to identify, understand and explain social thought in the field of environmental education. The most cited authors in the above mentioned researches are Denise Jodelet, Serge Moscovici, Marcos Reigota and Jean Claude Abric. In this brief review shows that predominate the researches with students, most used a qualitative methodology and interpretive approach. In the various studies analyzed the representations of the environment shows its importance in understanding the practice of environmental education. For the environmental education, it is essential to understand the ways in which social representations, are established in the deconstruction-construction, of the elements that make your everyday thinking. Considering the results of the studies reviewed, we can say that the environment is a complex social order, cultural and contextually determined.
\end{abstract}

Keywords: EDUCATION, ENVIRONMENT, RESEARCH, SOCIAL REPRESENTATIONS, MEXICO, BRAZIL

\footnotetext{
7 Doctor en Pedagogía por la Universidad Nacional

Autónoma de México (UNAM). Profesor e investigador de la Universidad Pedagógica Nacional, México.
}

Dirección electrónica: calixtoflores@hotmail.com

Artículo recibido: 9 de julio, 2012

Aprobado: 22 de noviembre, 2012 


\section{Introducción}

Las representaciones han sido empleadas en diversas disciplinas como la antropología, pedagogía, psicología o sociología, entre otras. Así, por ejemplo, se usa el término representaciones con diversos adjetivos como cognitivas, mentales o sociales; estas últimas han impactado significativamente la investigación en América Latina en tres campos: el educativo, el de la salud y el de la política, ya que develan las maneras en que los actores se representan ciertas ideas y establecen sus programas de acción.

En el campo educativo hay investigaciones que tienen en las representaciones sociales (en adelante RS) su fundamento teórico, ya que se reconoce la importancia para la educación de la producción social de representaciones (Piña y Cuevas, 2004).

Las RS y la educación ambiental tienen muchos aspectos en común, como por ejemplo, su análisis crítico del desarrollo de la sociedad. Ambas emergen en la década de los setenta, como una posición contestaria ante el discurso hegemónico de la modernidad.

En las RS se discriminan elementos y problemas del medio ambiente, estableciendo correspondencias con las prácticas educativas. La educación ambiental cuestiona las relaciones del ser humano con el medio ambiente.

Las RS, a diferencia de otras formas de conocimiento de sentido común, pueden constituir una guía para la planeación y la práctica de la educación ambiental. Y la educación ambiental, a diferencia de otros tipos de educación, se preocupa por la construcción de una conciencia crítica del desarrollo de la sociedad.

Este artículo conjuga la teoría de las RS con la educación ambiental; se presenta una exploración de investigaciones de las RS del medio ambiente en Brasil y México, con el propósito de observar los aportes de estas investigaciones para la educación ambiental.

\section{La teoría de las representaciones sociales}

Cada ser humano posee una gran cantidad de conocimientos y saberes, que tienen su origen en las experiencias de cada día. Para Juan Delval (2000), las representaciones se forman en las experiencias cotidianas de los sujetos, pueden referirse a grandes parcelas de la realidad, como el mundo físico, el mundo de la vida o el mundo social, o a aspectos muy limitados como el funcionamiento de un ecosistema o el ciclo de vida de un organismo. Estas representaciones le dan el sentido a la realidad y van cambiando, son dinámicas. 
En la psicología social, las representaciones se refieren a una estructura cognitiva que tiene, entre otras funciones, otorgarle un sentido al medio y servir de guía o plan a las conductas, al describir, clasificar y explicar la realidad. Las representaciones, de acuerdo con Javier Álvarez (2004), son sociales, porque permiten crear, entre las personas, redes de elaboración y transmisión de información.

Es de considerar que las RS se constituyen como una forma de conocimiento cotidiano y práctico que permite obtener una visión funcional del mundo; a partir de las RS los seres humanos dotan de sentido a las acciones cotidianas; por eso, pueden compartirse los significados entre quienes mantienen diversas relaciones interpersonales.

El carácter social de las representaciones orienta su estudio hacia el conocimiento colectivo y su vinculación con la práctica.

Las representaciones permiten a las personas conducirse ante los distintos estímulos del medio ambiente y, a su vez, a actuar sobre este. Cuando las representaciones se comparten en un grupo, estas adquieren el adjetivo de sociales, ya que se construyen en un proceso de actividad cultural, son un fenómeno histórico y social de la sociedad contemporánea.

A partir de estas representaciones, el ser humano determina sus intereses y expectativas, que lo impulsan a una interacción continua con los otros seres humanos y con su entorno, es así como se delimita y clasifica al medio ambiente.

Las RS corresponden a una teoría que explica la forma de ver el mundo. Comprende una serie de nociones acerca de un cierto objeto. Esta teoría critica las posturas clásicas de la psicología conductual en las que la relación sujeto-objeto está basada solo en la relación estímulo-respuesta, en cambio, afirma que en la relación entre el objeto y el sujeto existe una relación interdependiente.

Serge Moscovici, iniciador de la teoría de las RS, las define como

Sistemas de valores, ideas y prácticas que tienen una doble función: en primer lugar, establecer un orden que permita a los individuos orientarse en un mundo social y material y dominarlo; y, en segundo término permitir la comunicación entre los miembros de una comunidad, aportándoles un código para el intercambio social y un código para denominar y clasificar de manera inequívoca los distintos aspectos de su mundo y de su historia individual y grupal. (Moscovici, 1973, p. 13) 
Las RS se orientan hacia la comunicación, la comprensión y el dominio del entorno material y social. Son construcciones de los sujetos sobre un objeto, pero nunca reproducciones de ese objeto.

Para las RS son de igual importancia los aspectos cognitivos y los de constitución social de lo real. La teoría de las RS aún está en desarrollo, son un aporte teórico desde la psicología social, donde el peso de lo social no niega la importancia del individuo.

En relación con los otros, el individuo en situación, con un conjunto de vínculos que van conformando un grupo, hace posible la construcción de las RS, con las que confieren significados a los objetos y adquieren sentido. Las RS se construyen en colectivo y mantienen un fuerte vínculo con las prácticas que realizan los sujetos en este colectivo.

En tanto que fenómenos, las RS se presentarán bajo formas más o menos complejas: imágenes que condensan un conjunto de significados: sistemas de referencia que permiten interpretar lo que sucede, e incluso, dar un sentido a lo inesperado. Categorías que sirven para clasificar las circunstancias, los fenómenos y a los individuos con quienes se tiene algo que ver. Teorías que permiten establecer hechos sobre ellos. Y, a menudo, cuando se les comprende dentro de la realidad concreta de la vida social, las RS son todo ello junto. (Jodelet, 1986, p. 472)

Las RS tienen una naturaleza convencionalizadora y otra prescriptiva, con las cuales determinan y condicionan la experiencia de los sujetos. Emergen en las interacciones cotidianas de cada grupo social, Moscovici refiere tres condiciones para que aparezcan: la dispersión de la información, la focalización del sujeto individual y colectivo y la presión a la inferencia del objeto socialmente definido. Estas circunstancias hacen posible la determinación de la organización cognoscitiva de la representación.

La dispersión de información se refiere a los datos que posee el grupo social respecto a un objeto específico, la focalización cuando se delimita un objeto, que llama la atención al grupo, es diversa y casi siempre excluyente, y la presión de inferencia, en términos de Moscovici (1979), se refiere a las circunstancias y las relaciones sociales que existen en el individuo o del grupo social para que sean capaces en todo momento de estar en situación de responder. 


\section{La educación ambiental}

Los múltiples problemas ambientales que agobian a las sociedades contemporáneas evidencian la necesidad de una búsqueda constante de alternativas de solución. Estas se han dado principalmente con el desarrollo de nuevas tecnológicas que, por ejemplo, reducen la emisión de contaminantes o que emplean fuentes alternativas de energía, como la solar o la eólica. Sin embargo, se ha de considerar que no basta con buscar soluciones tecnológicas, resulta necesario prevenir los problemas ambientales con base en alternativas educativas.

La educación ambiental surge para buscar respuestas y generar nuevas preguntas en torno a las relaciones inequitativas de los seres humanos con el medio ambiente; sus finalidades son muy diversas, entre las que se encuentran el fomento de valores ambientales, que impulsen a los individuos y sociedades a evaluar el actual modelo de desarrollo económico y buscar mejores formas de relacionarse con el medio ambiente.

La educación ambiental resulta importante, porque permite generar valores ambientales para prevenir, mitigar o resolver los problemas ambientales, involucrando la participación activa y consciente de las diversas instituciones, grupos y actores sociales. Sin embargo, hay que tener presente que la educación tiene un papel limitado, cuando en diversos escenarios sociales se orienta a las personas hacia la búsqueda del placer, la acumulación de bienes materiales y al individualismo.

Revertir este modelo de ser humano requiere que la educación ambiental comprenda la generación de valores ambientales, así como comprender la esencia de las personas, que permita rescatar su dignidad y ofrecer diversas alternativas de desarrollo humano. Pero este no necesariamente implica la posesión de bienes materiales, sino vivir dignamente, aprender a tener una vida plena y ser respetado.

Los valores ambientales se pueden entender como el conjunto de cualidades que definen un ambiente como tal, incluidos los componentes, de acuerdo con el tipo de ambiente a que se refiera: natural, social o construido.

La educación ambiental contribuye a explicar la existencia y la vigencia de todo un mundo de producción humana que tiene importancia definitiva para la vida del planeta Tierra. La educación ambiental puede generar y mantener usos y creencias que impulsen el desarrollo de una cultura ambiental, y como consecuencia puede ser el medio para el logro de nuevas relaciones entre los seres humanos, y de estos con el medio ambiente. 
A la educación ambiental le interesa no solo explicar los problemas ambientales del medio natural, sino también el social y el transformado, en el que se manifiestan con toda claridad las distintas responsabilidades de los distintos sectores sociales. Estos problemas hacen evidente la necesidad de tomar decisiones y actuar sobre los problemas inmediatos, sin perder de vista las acciones a futuro. En este sentido, entre las finalidades de la educación ambiental se encuentran la de fomentar una conciencia ambiental comprometida con la realidad social, la de formar actitudes y valores congruentes con un estilo de vida que propicie el desarrollo de relaciones equitativas con el entorno natural y social. Como también fomentar una manera de observarse a sí mismo, con relación a la totalidad de acontecimientos que orientan nuestra forma de sentir y pensar, como ciudadanos de un país y habitantes de un único planeta.

Los objetivos de la educación ambiental son:

Mayor sensibilidad y conciencia sobre los problemas del medio ambiente; conocimiento del medo ambiente y sus problemas como una unidad totalizadora de la que el ser humano forma parte y debe hacerlo con responsabilidad crítica; desarrollar en los individuos un sentido ético-social ante los problemas del medio que los impulse a participar activamente en su protección y mejoramiento; desarrollo de las aptitudes necesarias para resolver problemas ambientales; impulsar la capacidad de evaluar las medidas y los programas de educación ambiental en función de los factores ecológicos, políticos, económicos, sociales, estéticos y educacionales; crear conciencia de la urgente necesidad de prestar atención a los problemas del medio ambiente, para asegurar que se adopten medidas adecuadas a su respecto. (Teitelbaum, 1978, p. 50)

A partir de estos objetivos, es necesario agregar entre las finalidades de la educación ambiental el desarrollo de una conciencia de que la humanidad forma una unidad totalizadora con el medio ambiente y sus problemas. Las sociedades humanas no están fuera del medio ambiente, ni son su complemento, están integradas al medio ambiente. El ser humano está a un paso de extinguir miles de especies de animales y plantas y de organismos de otros reinos, que implican necesariamente el deterioro de las condiciones del planeta para albergar la vida. Hay que recordar que la especie humana es un miembro más del reino animal y que el exterminio de las otras formas de vida es el inicio de su propia extinción. 
Todos somos parte de la biodiversidad, existe una interdependencia entre todas las formas de vida del planeta, como con la propia Tierra.

La educación ambiental puede fomentar un cambio en las representaciones con las que los individuos clasifican al medio ambiente, que impactan en su relación con el medio ambiente, y asignar un valor diferente a las otras formas de vida, con las que comparte los bienes del planeta.

En este punto y siguiendo a Víctor Manuel Toledo (2003) es necesario cuestionar y replantear tanto los métodos como las bases mismas de la teoría neoclásica y de la economía de mercado. La educación ambiental cuenta con los elementos necesarios para realizar un análisis crítico y propositivo de los fundamentos sociales del actual modelo económico y de sus implicaciones para el medio ambiente.

En el ámbito escolar la finalidad de la educación ambiental debe ser: “... responder a la necesidad de que los individuos y grupos sociales adquieran, con la actividad educativa, la capacidad de hacer frente, con actitud abierta, relativa, autónoma y negociadora, a los problemas que el medio socio-natural plantea" (Martínez, 2007, p. 7).

La educación escolarizada tiene así múltiples posibilidades para poder incidir en las representaciones que los niños construyen desde sus primeras etapas de vida.

La educación ambiental constituye una de las alternativas que genera cambios en la forma de pensar en los seres humanos, sin embargo, si esta no es planeada, ni definidos sus objetivos, puede reducirse a actividades remediales, que no llegan a lograr la concientización sobre los efectos de las formas de actuar sobre el medio ambiente.

La educación ambiental no es solo "conservar la naturaleza" o "concientizar a las personas" o "cambiar conductas", es todo ello y algo más

La educación ambiental ante la crisis económica y social que vive nuestra realidad representa una opción viable para promover cambios sociales, nuevos conocimientos, crear conciencia de participación, de equilibrio social y ecológico, de desarrollo sustentable y de comportamientos culturales que hagan real una sociedad más igualitaria y solidaria, con objetivos interrelacionados para moldear una nueva dimensión del ser humano (Martínez, 2007, p. 23).

La educación ambiental implica necesariamente considerar las relaciones entre los seres humanos en las distintas sociedades que conforman la aldea global. 
Ernesto Sábato (2000) describe que la tarea es más profunda y comprometida, es educar para cambiar la sociedad; es procurar que la toma de conciencia se oriente hacia un desarrollo humano que sea simultáneamente causa y efecto de la sustentabilidad y la responsabilidad global; por lo que se identifica con una educación total para la mejora de la calidad de vida y de sus entornos, asumiendo su caracterización como una práctica política, promotora de valores que inciten la transformación social, el pensamiento crítico y la acción emancipadora.

La educación ambiental se inscribe así dentro de un proceso estratégico que estimula la reconstrucción colectiva y la reapropiación subjetiva del saber (Leff, 2002). La educación ambiental hace énfasis en una idea que se basa en un proceso de concientización sobre la problemática ambiental, que debe guiarse hacia la reorientación de valores, actitudes y conductas de la sociedad, incrementando el respeto al equilibrio ecológico: “...la educación ambiental es un proceso permanente de formación y aprendizaje en el que el individuo, en interacción con la sociedad en la que vive, intenta conservar y mejorar el medio que lo rodea" (Ramírez, 1992, p. 18).

Este tipo de educación pretende formar una conciencia individual y colectiva sobre los problemas ecológicos, que logre trascender a una conciencia social, puesto que a partir de ello se pretende formar un bachiller que intervenga en las decisiones que favorezcan restaurar el equilibrio del medio ambiente.

La transformación social que busca la educación ambiental requiere la conformación de sujetos capaces de interpretar los procesos de socialización, de una manera personal de pensar y de apreciar el mundo, de relacionarse con la naturaleza, de verse a sí mismo y a los demás. Requiere, además, del conocimiento científico sobre las causas, consecuencias y alternativas, a la problemática ambiental, en la que se encuentra la sociedad presente. Por eso, son tan importantes los procesos de formación donde el individuo sea capaz de reflexionar sobre su entorno y buscar soluciones viables a partir de lo que está a su alcance hacer.

Para Enrique Leff, la educación ambiental conlleva la formación de nuevas actitudes, que implica:

educar para formar un pensamiento crítico, creativo y prospectivo, capaz de analizar las complejas relaciones entre procesos naturales y sociales, para actuar en el 
ambiente con una perspectiva global, pero diferenciada por las diversas condiciones naturales y culturales que lo definen. (Leff, 1994, p. 217)

La educación ambiental se vincula a un proceso de construcción y apropiación de conceptos que generan sentidos divergentes sobre la sustentabilidad, arraigados en la vida de cada persona y de cada comunidad. Por eso, no es independiente de las tensiones que surgen entre la subjetividad y la esfera pública, entre el potencial de lo real y las visiones utópicas, entre el conocimiento y la acción social.

\section{Las representaciones sociales del medio ambiente}

El medio ambiente inicialmente consideraba las condiciones, influencias o fuerzas que envuelven, influyen o modifican un complejo de factores climáticos, edáficos y bióticos que actúan sobre un organismo vivo o una comunidad ecológica y acaban por determinar su forma y sobrevivencia. Pero el medio ambiente tiene aspectos que implican no solo a la naturaleza. Una de las acepciones de ambiente de la Real Academia Española lo designa como el: conjunto de circunstancias culturales, económicas y sociales en que vive una persona o un grupo humano.

Algunos estudiosos del medio ambiente no consideran apropiado este término, pues no corresponde a la traducción de Environment al español. Argumentan que el término medio ambiente es una incongruencia lingüística con deficiencia de contenido, porque es como una redundancia que mediatiza y desvirtúa el concepto ambiente. Sin embargo, el término de medio ambiente, da la posibilidad de identificar distintas relaciones, de acuerdo con el medio ambiente al que se haga referencia, pues este puede aludir tanto a elementos naturales como sociales.

En este sentido, se pueden utilizar como sinónimos ambiente o medio ambiente, ya que en el término ambiente se pueden incluir a todos los sistemas posibles en los cuales se relacionan los organismos; por ende, existen diversos ambientes.

De otra parte, se puede considerar al medio ambiente como una categoría social, relativa a un modo de ver y pensar la realidad, porque el medio ambiente es resultado de un proceso de construcción social-cognitiva, en el que se incluyen las relaciones que se establecen entre los componentes que lo constituyen. 
El medio ambiente es integral, con distintos tipos de ambientes y diversas relaciones. Si se considera en el ambiente los distintos tipos de relaciones, se debe incluir a quienes las construyen: los seres humanos, y es cuando cobra sentido el contenido de medio ambiente. En el que las relaciones de los seres humanos con el medio ambiente permiten identificar no solo un ambiente natural, sino también el transformado y el social.

El modelo de desarrollo económico dominante privilegia la noción del medio ambiente como fuente de recursos naturales, deja fuera aspectos fundamentales para comprenderlo como una red de relaciones, en la que están presentes las relaciones entre los seres humanos.

El medio ambiente comprende relaciones entre todos los organismos, pero que se explicitan con referencia a los seres humanos, quienes pueden construir diferentes tipos de relaciones.

El ser humano es un ser con intereses, expectativas y necesidades, que lo impulsan a una interacción continua con los otros seres humanos y con su entorno, es así como se delimita y clasifica el medio ambiente. El desarrollo de la humanidad ha propiciado que estos límites sean cambiantes, de acuerdo con la posición del ser humano.

Las características del medio ambiente dependen de las formas en que se da esta relación dialéctica, de la desconstrucción-construcción de tal relación de mutua determinación y transformación.

A partir de estas consideraciones, se debe comprender al medio ambiente como una relación dialéctica entre las leyes ecológicas (en su sentido biológico) y las leyes sociohistóricas que definen su estructuración (Jiménez, 1997). En este sentido, el medio ambiente es una construcción cultural:

En consecuencia, dicho conocimiento emerge desde múltiples saberes en confluencia con modelos de desarrollo, aspectos económicos, políticos, ecológicos y sociales, que están asociados con los cambios ambientales y que, a su vez, dan lugar a incorporar los principios de diversidad cultural, sustentabilidad ecológica, equidad social y solidaridad transgeneracional. (León, Vallejo, Parra y Obregoso, 2010, p. 3)

El medio ambiente es comunicado socialmente de diversas formas, debido a que es empleado y entendido de acuerdo con las representaciones que poseen los individuos. Esta comunicación, a partir del conocimiento cotidiano, da lugar a las RS. 
Marcos Reigota (1990) clasifica a las RS del medio ambiente en: antropocéntricas, globalizantes y naturalistas. Las RS antropocéntricas toman en cuenta los bienes y productos que benefician al ser humano, las relaciones con el medio ambiente al buscar la satisfacción de sus necesidades; las RS naturalistas centran su atención en los elementos de la naturaleza, se caracterizan por ubicar al medio ambiente natural como externo al ser humano. Las RS globalizantes plantean a la naturaleza y a la sociedad como procesos continuos y mutuamente interdependientes que conducen hacia la construcción de nuevos niveles de complejidad y diversidad.

\section{Exploración de investigaciones de las representaciones sociales del medio ambiente}

Al revisar investigaciones en el campo de la educación ambiental es posible detectar en varias de ellas el empleo de las RS del medio ambiente. La teoría de las RS es joven y se encuentra en proceso de formación; paralelamente, emerge la educación ambiental en la década de los setentas del siglo anterior.

Las investigaciones de las RS del medio ambiente, vinculadas con la educación ambiental, corresponden a la investigación educativa, no a la investigación en la psicología ambiental. En la literatura es común encontrar investigaciones del medio ambiente o de algún aspecto de este que no tienen entre sus propósitos la vinculación de sus resultados con la educación ambiental.

Las RS ocupan un papel importante en la investigación educativa porque tienen repercusiones en la producción cognitiva de los sujetos; independientemente de su origen, las RS permiten la conceptualización de lo real, a partir de la activación del conocimiento previo.

Las investigaciones de las RS del medio ambiente en el ámbito educativo han estudiado, principalmente, la forma de pensar de los profesores y alumnos, quienes son los principales actores del hecho educativo.

En este escrito se hace una exploración de algunas de las investigaciones que emplean las RS del medio ambiente en Brasil y en México, en el campo de la educación ambiental. Se eligieron estos dos países, porque en Latinoamérica, en Brasil y en México, se identifican numerosas investigaciones en educación ambiental, que utilizan la teoría de las RS como marco teórico y metodológico. En Brasil, con los trabajos de Marcos Reigota 
(1990), se dio inicio a la línea de investigación en RS del medio ambiente y su vínculo con la educación ambiental. Y en México es creciente el empleo de la RS en los estudios referidos a la educación ambiental, como se observa en los programas de doctorado en las Universidades Federal de Sao Carlos, Pontifícia Universidade Católica de São Paulo Universidade Federal do Rio de Janeiro y Universidade de Sorocaba de Brasil, y en la Benemérita Universidad Autónoma de Puebla, Universidad Pedagógica Nacional, Universidad Nacional Autónoma de México y Universidad Veracruzana de México. En estas universidades es posible encontrar tesis de doctorado en educación ambiental que utilizan la teoría de las RS.

\subsection{Exploración de investigaciones en representaciones sociales del medio ambiente en Brasil}

Marcos Reigota (1990), con la investigación "Les représentations sociales de l'environnement et les pratiques pédagogiques quotidiennes des professeurs de Sciences a São Paulo-Brésil”, ["Las representaciones sociales del medio ambiente y las prácticas pedagógicas cotidianas de los profesores de ciencias de Sao Paulo Brasil"], inicia una línea de investigación que vincula a las RS con la educación ambiental. Reigota clasifica a las RS en tres grandes tipos: naturalistas, globalizantes y antropocéntricas.

Angela Arruda (1998) realiza la investigación "O ambiente natural e seus habitantes no imaginário brasileiro:negociando a diferença”, [“Lo que piensa el brasileño sobre el medio ambiente, el desarrollo y la sustentabilidad"], en la que utiliza RS como sustento teórico; entre los resultados plantea que en las RS de los grupos de la sociedad brasileña aparecen aspectos detectados en el imaginario sobre el ambiente natural, identifican aspectos de la realidad presente e incorporan el temor que despierta la crisis ambiental. Las principales categorías utilizadas son la herencia cultural, la tropicalidad, cordialidad/solidaridad.

Por su lado, Eneida Maria Molfi Goya (2000) en su trabajo "Desconstrucción de las representaciones sobre el medio ambiente y la educación ambiental" investiga las RS que poseen maestros de educación superior y encuentra que estas son muy parecidas, representan al medio ambiente como un gran ecosistema, un lugar distante, frágil y desprotegido. Los participantes en la investigación, a través de lecturas y discusiones de textos, lograron identificar sus RS y desconstruirlas posteriormente. 
Rosana L. Ferreira (2002) en la investigación "Representaciones sociales de medio ambiente y educación ambiental de docentes universitarios (as)" analiza el discurso oral y escrito de 19 maestros de instituciones de educación superior, que imparten algún curso relacionado con la educación ambiental. Además, utiliza las categorías propuestas por Reigota (1990), y descubre que las representaciones del medio ambiente y educación ambiental son muy heterogéneas, varían entre naturalistas y globalizantes, aunque predominan estas últimas.

Christine Storey (2003) en la investigación "Representações sociais e meio ambiente: participação de um grupo de mulheres no planejamento de uma intervenção de educação ambiental popular urbana em Manaus, Amazonas", ["Representaciones sociales y medio ambiente: participación de un grupo de mujeres en la planeación de una intervención de educación ambiental popular urbana en Manaus, Amazonas"], interpreta las necesidades percibidas y las relaciones ambientales de un grupo de 10 mujeres, por medio de la teoría de las RS. Los resultados revelan que las RS influyen en la visión del mundo y consecuentemente en sus actos; encuentra que estas RS son construidas principalmente por la influencia de cinco factores: herencia cultural, la identidad, medios de comunicación, religión y contexto. Identifica representaciones sociales sobre el medio natural, humano y construido.

Carla Fernanda Bernardino, Laísa Maria Freire, Alexandre Ferreira y Reinaldo Ruiz (2007) en el estudio "Análise das representações sociais sobre meio ambiente de técnicos e professores das secretarias de educação e meio ambiente de municípios da Bacia de Campos", ["Análisis de las representaciones sociales sobre el medio ambiente de técnicos y profesores de la Secretaria de Educación y Medio Ambiente de los municipios de Bacia de Campos"], en el que participaron 36 técnicos y profesores de la secretaría de educación y medio ambiente, de 13 municipios de la región, encontraron el predominio de las representaciones sociales naturalistas. Estos resultados permiten comprender la orientación que le imprimen a las acciones que realizan en educación ambiental.

André Luis de Oliveira, Ana Tiyomi Obara y Maria Aparecida Rodrigues (2007) en la investigación "Educação ambiental: concepções e práticas de professores de ciências do ensino fundamental", ["Educación ambiental: concepciones y prácticas de profesores de ciencias de enseñanza elemental"], en la que participaron once profesores de ciencias de educación primaria, de cinco escuelas públicas; identifican la existencia de representaciones 
antropocéntricas relacionadas con la ética antropocéntrica humanista y el pensamiento cartesiano, que coloca al ser humano fuera de la naturaleza. Y Pedro Pablo Saleme, Alice Pereira de Faria y Jorge Luiz de Góes Pereira (2011) en el estudio "Representações sociais de Meio Ambiente e educação ambiental nas escolas públicas do meio rural e urbano de Teófilo Otoni/MG", ["Representaciones sociales del medio ambiente y educación ambiental en escuelas públicas del medio rural y urbano de Teófilo Otoni/MG"], analizan las RS del medio ambiente de los profesores y los estudiantes de primaria en escuelas públicas de zonas urbanas y rurales Teófilo Otoni-MG, y su relación con proyectos de educación ambiental desarrollados en estas escuelas; describen el predominio de las representaciones antropocéntricas, sobre todo entre estudiantes de las escuelas rurales, frente a los de colegios urbanos.

En el conjunto de estudios analizados se observa el predominio de las RS antropocéntricas y el estudio en diversos contextos y poblaciones. La existencia de una diversidad de representaciones, habla de la existencia de una práctica asumida de la educación ambiental. En las investigaciones descritas se observa el vínculo entre las RS del medio ambiente y la educación ambiental.

\subsection{Exploración de investigaciones en representaciones sociales del medio ambiente en México}

Entre las investigaciones realizadas en México está la de Antonio Fernández Crispín, Javier Benayas del Álamo y Clara Barroso (2005), quienes con la investigación "Social representation of the way to interact with environment of the elementary school teachers of the Puebla's municipality (Mexico), [“Las representaciones sociales de la intercacción con el medio ambiente de los profesores de educación primaria del municipio de Puebla (México)"], determinan el concepto de educación ambiental y modelo civilizatorio de los maestros de educación primaria, del municipio de Puebla; en dicho análisis se demuestra que los niños y profesores tienen RS de los problemas ambientales y poseen construidos los themata, que tocan de forma superficial los problemas del medio ambiente. En la investigación de Raúl Calixto (2009), realizada con estudiantes de la Licenciatura en Educación Primaria, se muestra que existen más RS naturalistas (asociadas con la naturaleza y la preservación) que los otros tipos de RS como globalizantes, antropocéntricas utilitarias, antropocéntricas 
pactuadas y antropocéntricas culturales. Se observa una relación entre las RS del medio y la práctica de la educación ambiental.

Por su parte, Esperanza Terrón (2010) en la investigación "Educación ambiental. Representaciones sociales y sus implicaciones educativas" plantea que las RS de los actores educativos son construidas a través de una red de relaciones y prácticas comunicativas que dan distintos matices a la práctica de la educación ambiental. En el estudio "La educación ambiental en la escuela secundaria pública: su incorporación en Saltillo, Coahuila", de Rosa Valdez (2011) aborda las RS de los profesores y alumnos acerca del medio ambiente y de la educación ambiental; la autora reporta solo las RS de los alumnos y encuentra que son preferentemente naturalistas, es decir, que evidencian los elementos naturales y los seres vivos.

En el trabajo "Educación ambiental y uso del agua" Raúl Calixto (2011) evidencia la importancia de formar en educación ambiental entre los futuros docentes de educación básica. Se observa que las representaciones de los profesores en formación están más vinculadas con la información que proporciona la televisión, que con los contenidos que se encuentran de los programas de estudio; las RS del uso del agua están asociadas con el desperdicio, identifican el agua como un recurso limitado, por lo que es importante la educación ambiental.

En México, a diferencia de Brasil, las investigaciones de las RS del medio ambiente, vinculadas a la educación ambiental, iniciaron 15 años después; se identifican más las RS naturalistas, además, es más frecuente el desarrollo de las investigaciones en la educación escolarizada. Al igual que las investigaciones realizadas en Brasil, se observa que existen distintos tipos de RS, que orientan, al mismo tiempo, distintos tipos de prácticas en educación ambiental.

La exploración de las investigaciones realizadas en Brasil y en México muestra la pertinencia del empleo de la teoría de las RS, para identificar, comprender y explicar el pensamiento social en el campo de la educación ambiental. Los autores más citados en las investigaciones anteriormente referidas son Serge Moscovici (1979), Denise Jodelet (1986), Marcos Reigota (1990) y Jean Claude Abric (2001). En esta breve revisión se observa que predominan las investigaciones realizadas con estudiantes, la mayoría utiliza una metodología cualitativa y un enfoque interpretativo. Se puede ubicar un predominio del enfoque procesual de las RS, enfoque que privilegia el análisis del discurso de los sujetos. 


\section{Conclusiones}

En este artículo, se observa la existencia de RS del medio ambiente, representaciones que se traducen en prácticas específicas en torno a la educación ambiental. El reconocimiento de la existencia de distintas representaciones permite configurar el pensamiento sobre el medio ambiente, y explicar en el caso de los profesores de Brasil y México, el predominio de las corrientes naturalista y antropocéntrica en la educación ambiental.

En educación ambiental existen varias corrientes, cada una prioriza uno u otro aspecto del medio ambiente. Las RS del medio ambiente se vinculan a determinadas corrientes en educación ambiental. Lucie Sauvé (2004) identifica las siguientes corrientes de la educación ambiental: naturalista, conservacionista/recursista, resolutiva, sistémica, científica, humanista, moral/ética, holística, bioregionalista, práxica, crítica, feminista, etnográfica, ecoeducación y sostenibilidad/sustentabilidad. Sauvé, hace incapié que estas corrientes no son mutuamente excluyentes.

Las RS del medio ambiente corresponden a un tipo de conocimiento de sentido común -con elementos de la ciencia- que se construye en la vida cotidiana en grupos referenciados y que atienden a un momento histórico. En este sentido, es posible la reconstrucción de las $\mathrm{RS}$, incorporando nuevos elementos a través de la educación.

Para abordar la educación ambiental, resulta fundamental comprender las formas en que se establecen las RS, en la desconstrucción-construcción de los elementos que forman su pensamiento cotidiano.

Considerando los resultados de las investigaciones revisadas, se puede afirmar que el medio ambiente es un objeto social complejo, cultural y contextualmente determinado. Rebasa las delimitaciones conceptuales que desde la biología o la geografía se han establecido. El medio ambiente es un producto social, que refleja el sentido que las personas le proporcionan en un momento específico.

A partir de que algunos sectores de la sociedad tomaron conciencia de los efectos dañinos de la actividad industrial en la salud humana aumentó la preocupación por el mejoramiento de la calidad del medio ambiente. En este contexto es hasta mediados del siglo XIX cuando emergen personas, grupos y organizaciones que intentan conservar en las mejores condiciones el medio ambiente natural. Por su parte, es en el siglo XX cuando la 
antropología, arqueología y la ecología muestran evidencias científicas de la interdependencia de la diversidad cultural humana y la diversidad biológica.

En las sociedades occidentales se ha privilegiado la idea de que la naturaleza es un bien inagotable, gratuito y externo, con lo que se fomenta el desarrollo de representaciones antropocéntricas del medio ambiente. Sin embargo, en los últimos años se ha hecho evidente que la biosfera es un elemento perecedero debido a su gran fragilidad y corre el riesgo de desaparecer, lo que afectaría a cada uno de los seres vivos que coexisten en el planeta Tierra.

La educación ambiental tiene que incorporar estos aspectos en sus propuestas, se hace necesario tomar conciencia de la importancia de la preservación del medio ambiente para la continuación de la vida como se conoce y se ha disfrutado. El predominio de las representaciones antropocéntricas o naturalistas del medio ambiente han favorecido la generación de múltiples problemas ambientales, ya que al socializarse y compartirse en la vida cotidiana, estos problemas ya no se observan como tales. Estas representaciones pasan del plano individual al social y constituyen comunidades que comparten una forma similar de observar al medio ambiente. En este sentido, la educación ambiental puede contribuir a la reconstrucción de estas representaciones, al favorecer la incorporación del ser humano como parte del medio, e integrar los aspectos naturales, sociales y culturales.

Al realizar una revisión de algunas investigaciones identificadas con las RS del medio ambiente en Brasil y México destaca el hecho de que sus resultados son coincidentes, a pesar de que sean distintas culturas y lenguajes, así como diferentes grupos de población y niveles educativos. Las poblaciones estudiadas manejan información semejante y poseen representaciones muy parecidas.

En este escrito, se resalta la idea de que las RS del medio ambiente comprenden elementos que caracterizan a la educación ambiental. En estas representaciones es posible identificar elementos presentes en el pensamiento cotidiano de los sujetos sobre el medio ambiente. Las RS adquieren una connotación de su origen que implica el compartirlas en un grupo, en las que se hace evidente que son construcciones de las personas sobre un objeto, pero nunca reproducciones de ese objeto.

En el conjunto de investigaciones analizadas resulta significativo que el peso de lo social no niega la importancia del individuo y resultan valiosas para comprender el pensamiento de las personas. 
En las diversas investigaciones analizadas sobre las RS del medio ambiente resalta la importancia de esta teoría para comprender la práctica de la educación ambiental; además, se observan elementos que las distinguen entre sí, cuando hacen énfasis en los componentes de la naturaleza, en la integración de lo natural con lo social o en el predominio del beneficio para el género humano. Pero, también, en todas las RS se hacen evidentes las asociaciones que se establecen con la educación ambiental.

\section{Referencias}

Abric, Jean Claude. (2001). Prácticas sociales y representaciones. México: Ediciones Coyoacán.

Álvarez, Javier. (2004). El contexto social y teórico del surgimiento de la teoría de las representaciones sociales. En Eulogio Romero (ed.), Representaciones sociales. Atisbos y cavilaciones del devenir de cuatro décadas (pp. 29-53). México: Benemérita Universidad de Puebla.

Arruda, Angela. (1998). O ambiente natural e seus habitantes no imaginário brasileiro: negociando a diferença. En Arruda A. (org.) Representando a alteridade (pp. 17-46). Río de Janeiro, Brasil: Vocez.

Bernardino, Carla Fernanda; Freire, Laísa Maria; Ferreira, Alexandre y Ruiz, Reinaldo. (2007). Análise das representações sociais sobre meio ambiente de técnicos e professores das secretarias de educação e meio ambiente de municípios da Bacia de Campos. Atas do VI Encontro Nacional de Pesquisa em Educação em Ciências, sp. Belo Horizonte, Brasil :Florianópolis.

Calixto, Raúl. (2009). Representaciones sociales del medio ambiente en los estudiantes de la Licenciatura en Educación Primaria. México: UPN.

Calixto, Raúl. (2011). Educación ambiental y uso del agua. En memoria electrónica del XI Congreso Nacional de Investigación Educativa. México: COMIE. Recuperado de http://digitalacademico.ajusco.upn.mx:8080/upn/bitstream/123456789/47/1/0169.pdf

Delval, Juan (2000). Aprender en la vida y en la escuela. Madrid, España: Morata.

De Oliveira, André Luis; Tiyomi Obara, Ana e Rodrigues, Maria Aparecida. (2007). Educação ambiental: concepções e práticas de professores de ciências do ensino fundamental. Revista Electrónica de Enseñanza de las Ciencias, 6(3), 471-495.

Fernández-Crispín, Antonio; Benayas del Álamo, Javier y Clara Barroso. (2005). Social representation of the way to interact with environment of the elementary school teachers of the Puebla's municipality (Mexico). International Journal of Environment and Sustainable Development, 4(2), 140-153. 
Ferreira, Rosana. (2002). Representaciones sociales de medio ambiente y educación ambiental de docentes universitarios. Tópicos en Educación Ambiental, 4(10), 22-36.

Goya, Eneida Maria Molfi. (2000). Desconstrucción de las representaciones sobre el medio ambiente y la educación ambiental. Tópicos en educación ambiental, 4(02), 33-40.

Jiménez, Ma. del Pilar. (1997). Las representaciones y sus implicaciones. En Teresa Wuest et al., Pensamiento universitario. Formación, representaciones, ética y valores, 87 (pp.15-25). México: CESU-UNAM.

Jodelet, Denise. (1986). La representación social: fenómenos, conceptos y teoría. En Serge Moscovici, Psicología Social II (pp. 469-534). Barcelona: Ediciones Paidós.

Leff, Enrique (comp.). (1994). Ciencias Sociales y Formación Ambiental. Barcelona, España: Gedisa.

Leff Enrique; Ezcurra, Ezequiel; Pisanty, Irene y Romero Lankao, Patricia (comp.). (2002). La transición hacia el desarrollo sustentable, Perspectivas de América Latina y el Caribe. México: Instituto Nacional de Ecología, Universidad Autónoma Metropolitana y Programa de Naciones Unidas para el Medio Ambiente.

León, Jennifer; Vallejo, Catalina; Parra, Adriana y Obregoso, Yolima. (2010). Clasificación múltiple de ítems y las representaciones sociales sobre ambiente en profesores rurales. Revista Electrónica, Actualidades investigativas en educación, 10(2), 1-26. Recuperado de http://revista.inie.ucr.ac.cr/uploads/tx magazine/items.pdf

Martínez, Roger. (2007). Aspectos políticos en educación ambiental. Revista Electrónica Actualidades Investigativas en Educación, 7(3), 1-25. Recuperado de http://redalyc.uaemex.mx/pdf/447/44770318.pdf

Moscovici, Serge. (1973). Prólogo a C. Herzlich. En Health and IIIness (pp. 13-26). London: Academia Press.

Moscovici, Serge. (1979). El psicoanálisis, su imagen y su público. Buenos Aires: Huemul. (Trabajo original publicado en 1961).

Piña Osorio, Juan Manuel y Cuevas Cagija, Yazmín. (2004). La teoría de las representaciones sociales. Su uso en la investigación educativa en México. Perfiles educativos, 26(105-106), 102-124.

Reigota, Marcos. (1990). Les représentations sociales de l'environnement et les pratiques pédagogiques quotidiennes des professeurs de Sciences a São Paulo-Brésil. (Tese de doutorado em pedagogia da biología), Louvain-laNeuve, Université Catholique de Louvain, Belgique.

Ramírez, Axel. (1992). El papel estratégico de la educación en el proceso de desarrollo sustentable. En Memoria del Foro Internacional para la Incorporación de la Dimensión 
Ambiental en la Educación Media Superior, pp. 17-25, Aguascalientes, México, Organización de Estados Americanos/Secretaría de Educación Pública.

Sábato, Ernesto. (2000). La resistencia. Barcelona: Seix Barral.

Saleme, Pedro Paulo; Pereira de Faria, Alice y de Góes Pereira, Jorge Luiz. (2011). Representações sociais de Meio Ambiente e educação ambiental nas escolas públicas do meio rural e urbano de Teófilo Otoni/MG. Revista Brasileira de Educação Ambiental (REVBEA), 6,(1), 35-40.

Sauvé, Lucie. (2004). Una cartografía de corientes en educación ambiental: In Miche’le, Sato y Carvalho Isabel (orgs.), A pesquisa em educacao ambiental: cartografias de uma identidade narrativa em formacao. Alegre, Brasil: Université du Québec à Montréal. Catadra de investigación de Canadá en educación ambiental, en: http://www.ambiente.gov.ar/infotecaea/descargas/sauve01.pdf

Storey, Christine. (2003). Representações sociais e meio ambiente: participação de um grupo de mulheres no planejamento de uma intervenção de educação ambiental popular urbana em Manaus, Amazonas. (Tese Doutorado), Universidade Federal de Sao Carlos, Brasil.

Teitelbaum, Alejandro. (1978). El papel de la educación ambiental en América Latina. Paris: UNESCO.

Toledo Víctor Manuel. (2003). El desafío planetario: integrar lo urbano, lo rural y lo natural. En Martí Boada y Víctor Manuel Toledo, El planeta, nuestro cuerpo, La ciencia para todos, 194 (pp. 111-200). México: Fondo de Cultura Económica.

Terrón, Esperanza. (2010). Educación ambiental. Representaciones sociales y sus implicaciones educativas. México: UPN.

Valdez, Rosa Elvira. (2011). La educación ambiental en la escuela secundaria pública: Su incorporación en Saltillo, Coahuila. En memoria electrónica del XI Congreso Nacional de Investigación Educativa, México: COMIE. Recuperado de http://www.comie.org.mx/congreso/memoriaelectronica/v11/docs/area 03/0914.pdf 